\title{
Optimum Resource Allocation in Orthogonal Frequency Division Multiplexing Communication System using Fuzzy Rule Base System and Particle Swarm Optimization and Comparison with Conventional other Techniques
}

\author{
Farhana Mustafa ${ }^{a}$, Padma Lohiya ${ }^{\text {b }}$ \\ ${ }^{a}$ Dept. of Electronics and Telecommunication, D. Y. Patil College of Engineering, Pune-41 1044, India \\ ${ }^{b}$ Asst. Prof, Dept. of Electronics and Telecommunication, D. Y. Patil College of Engineering, Pune-411044, \\ India
}

\begin{abstract}
OFDM is a technique that is chosen for high data rate communication and is important for 4th generation communication systems. To provide best possible quality of service, intelligent allocation of resources such as power, bandwidths are necessary. Various algorithms have been proposed for allocation of resources. Fuzzy Rule Based System (FRBS) and Particle Swarm Optimization (PSO) algorithm are used for optimization of code rate, modulation and power. FRBS is used to choose the best possible modulation and code rate and PSO is used for better power allocation.
\end{abstract}

Index Terms: OFDM, Fuzzy rule base system, Particle swarm optimization, Resource allocation.

(C) 2015 Published by MECS Publisher. Selection and/or peer review under responsibility of the Research Association of Modern Education and Computer Science

\section{Introduction}

Orthogonal Frequency Division Multiplexing (OFDM), offers a considerable high spectral efficiency multipath delay spread tolerance, immunity to frequency selective fading channels and power efficiency [1], [2]. As a result, OFDM has been chosen for high data rate communication and has been widely deployed in many wireless communication standard such as based mobile worldwide interoperability for microwave access (mobile WIMAX), 3GPP long term evolution (LTE) based on OFDM access technology. In OFDM every sub channel experiences a different channel condition so the use of same modulation and code rate may not be suitable for all subcarriers. Also, flat power would not be beneficial since sub-channels may need different

* Corresponding author

E-mail address: farhanabhatt23@gmail.com 
power. This situation demands adaptive resource allocations for an optimum utilization. The optimal power allocation and user selection solution was derived based on Lagrange dual decomposition proposed by [3] for maximizing the system energy efficiency. A low complexity algorithm for proportional resource allocation in Orthogonal Frequency Division Multiple Access (OFDMA) system was proposed in [4], where linear method and root finding algorithm were used to allocate power and data rates to users. A gradient based solution was proposed by [5], for downlink OFDM wireless systems and a 96.6\% utility was achieved. A Genetic Algorithm based adaptive resource allocation scheme was proposed by Reddy [6] to increase the user data rate where water-filling principle was used as a fitness function. The water filling theorem is based on a continuous relationship between the allocated power and the achievable capacity. Another paper with adaptive resource allocation based on modified genetic algorithm (GA) and particle swarm optimization (PSO) for multiuser OFDM system was proposed in [7]. In this paper it has shown that multi objective particle swarm optimization (MOPSO) is better than 3GPP LTE and NSGA II Algorithm.

This paper consists of four sections. Section II consist of Multi Modulation OFDM system where QAM modulation is taken in consideration with $\mathrm{M}=4,16,32,64,128,256$ Section III consist of FRBS and PSO algorithm. Section IV describes the simulation and results for OFDM system. Section V concludes the paper.

\section{Orthogonal Frequency Division Multiplexing}

The system model considered is OFDM equivalent baseband model with $\mathrm{N}$ number of subcarriers. It is assumed that complete channel state information (CSI) is known at receiver. The frequency domain representation of system is given by

$$
r_{k}=h_{k} \sqrt{P_{k}} X_{k}+Z_{k} ; k=1,2,3 \ldots N
$$

Where $\mathrm{r}_{\mathrm{k}}, \mathrm{h}_{\mathrm{k}}, \mathrm{P}_{\mathrm{k}}, \mathrm{Z}_{\mathrm{k}}$ and $\mathrm{X}_{\mathrm{k}}$ represents amplitude, transmit symbol and the Gaussian noise of sub carrier $\mathrm{k}$ respectively. It is assumed that signal transmitted on the $\mathrm{k}_{\mathrm{th}}$ subcarrier is propagated over Rayleigh at fade channel and each subcarrier faces a different amount of fading independent of each other. This can be given mathematically as

$$
H_{k}=\alpha e^{j \theta}
$$

where $h_{k}$ is Rayleigh distributed random variable of kth subcarrier. The proposed adaptation model is given in Fig 1

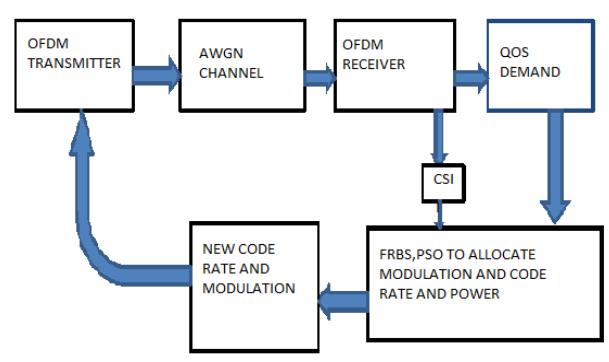

Fig.1. OFDM Model using Fuzzy Rule Based System and PSO 


\section{Code Rate}

Performance of standard modulation and codes being used in IEEE $802.11 \mathrm{n} 1 \mathrm{~g} / \mathrm{b}$ are analyzed in terms of bit error rate (BER) and SNR. Calculation of coding scheme, modulation scheme and channel is estimated. The code rate are taken from the set $\mathrm{C}$

$$
C=\left\{\frac{1}{4}, \frac{1}{3}, \frac{1}{2}, \frac{2}{3}, \frac{3}{4}\right\}
$$

Modulation symbol are taken from

$$
M=\{2,4,8,16,32,64,128\}
$$

Total number of MCPS can be given by

$$
P=C \times M
$$

\section{Fuzzy Rule Base System And Particle Swarm Optimization}

To maximize the data rate FRBS is used for optimum selection of modulation code pair (MCP) per subcarrier based upon received SNR and QoS.

\subsection{The steps involved in creation of FRBS are described below}

\section{i. Data Acquisition}

The information about SNR and BER obtained from Coded Modulation can be expressed as "for a given SNR and specific QOS which modulation code pair can be used.

\section{ii. Rule Formulation}

Rules for every pair are obtained by the appropriate fuzzy set used.

iii. Elimination of conflicting rule

This is used for eliminating conflicting rules.eg If there are two different pairs with same throughput like [2, $1 / 2]$ and $[4,1 / 4]$, both have same throughput i.e. $1 \times 1 / 2=0.5$. Thus $[2,1 / 2]$ is chosen since it have less modulation/ demodulation, coding/ decoding cost.

\section{iv. Completion of Look Up Table}

If complete numbers of IO pairs are not present, then those parts are filled by heuristic or expert knowledge. Example a modulation code pairs is suggested by rule for a certain SNR and QOS. Then that rule can also be used for slightly above SNR and poor QOS.

v. Fuzzy rule base creation 
The input output pair for design of FRBS is of the form

$$
\left(x_{1}^{s}, x_{2}^{s}, y_{3}^{s}\right) ; s=1,2,3, \ldots S
$$

Where $\mathrm{x}_{1}{ }^{\mathrm{s}}$ represents received SNR, $\mathrm{x}_{2}^{\mathrm{s}}$ represents BER (QOS) and $\mathrm{y}_{3}{ }^{\mathrm{s}}$ represents the output MCP.

\subsection{Fuzzy set:}

Input for Fuzzy inference system is given as SNR and BER or minus log BER. Relation between BER and MLBER is equal

$$
\begin{gathered}
\text { MLBER }=-\log (B E R) \\
B E R=10^{-q} \\
M L B E R=-\log \left(10^{-q}\right)=q
\end{gathered}
$$

There will be one output as MCP

\subsection{Membership function}

Membership function used in FIS (fuzzy inference system) is triangular. Triangular membership function is simple to implement as well as calculation of arithmetic operation is easier than bell, Sigmoidal and Gaussian. In FIS system AND is used for MIN and OR as MAX.

\subsection{Rule base}

7 sets of SNR and 10 sets MLBER are taken. Total number of rules taken is 70 that will be used in FIS system.

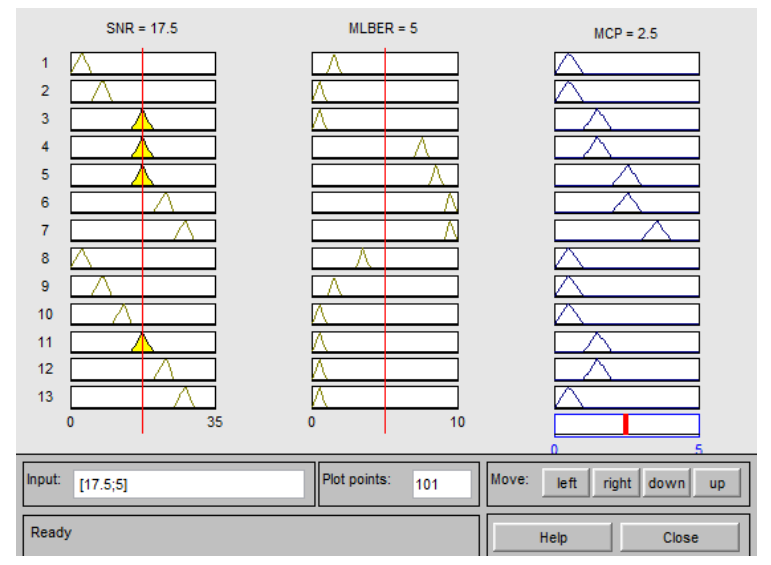

Fig.2. Rule Formation MATLAB View 


\subsection{DE- FUZZIFIER}

Standard Center Average Defuzzifier (CAD) is used for defuzzification. CAD is to perform a linear combination over the computed weights at the fuzzy inference engine and then modify this combination by novelization. CAD provides continuity and homogeneity and has less computational complexity.

\section{Particle Swarm Optimization}

PSO is a stochastic optimization technique developed by Eberhart and Kennedy inspired by the social behavior of flocks of bird. Each particle is represented by a position and velocity vector. Let dimensions of position and velocity vectors are defined by the number of decision variables in optimization problem. Soft PSO has been utilized for finding the optimum power vector for all the sub carriers depending upon the channel conditions and their QOS demand. Each sub-channel have different channel condition so different channel should have different power allocation depending upon the channel condition. Power allocation will be done with the help of PSO

Let $\bar{x}_{i}(t)$ represents the position particle $p_{i}$ at time t. The position of $p_{i}$ is then changed by adding a velocity $\bar{x}_{i}(t)$. That is

$$
\bar{x}_{i}(t)=\bar{x}_{i}(t-1)+\bar{v}_{i}(t)
$$

Each particle know its best position (p-best) and global best(g-best).Thus the particle will tend to attain its gbest at final iteration.

$$
\bar{v}_{i}(t)=\bar{v}_{i}(t-1)+C_{1} r_{1}\left(\bar{x}_{\text {pbest }}-\bar{x}(t)\right)+C_{2} r_{2}\left(\bar{x}_{\text {gbest }}-\bar{x}(t)\right)
$$

Where $\mathrm{C}_{1}$ and $\mathrm{C}_{2}$ are constants and is normally equal to 2.0. $\mathrm{r}_{2}$ and $\mathrm{r}_{1}$ are random variables.

The fitness block for local and global particles is given in fig 5

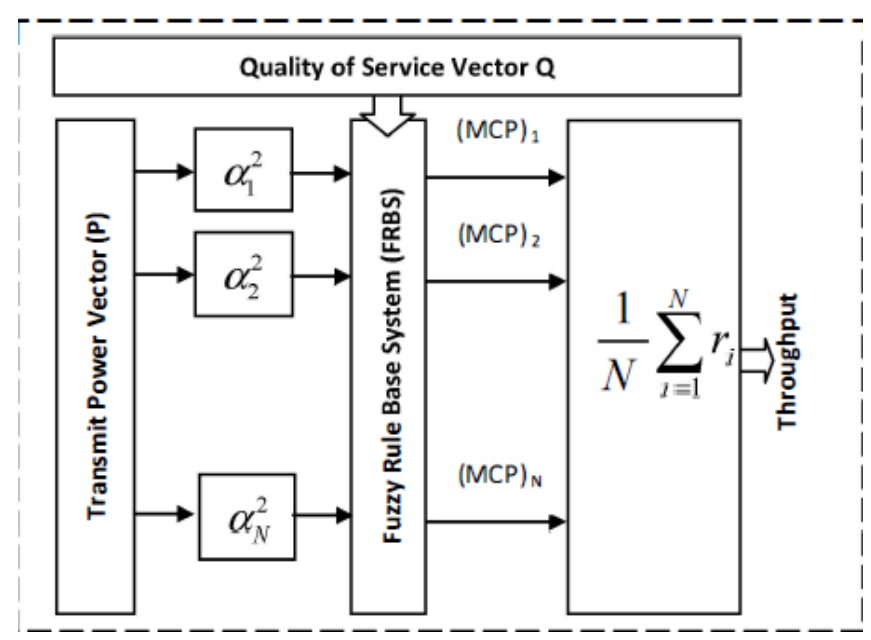

Fig.3. Fitness Block 


\section{Proposed Methodology}

First the system model of an OFDM is developed using MATLAB. Then the BER and SNR value is calculated using the graph that is simulated from system model. Then the BER and SNR value is given as input to the FIS system and throughput and modulation as output. FRBS give the best modulation that will have the highest throughput. After using FRBS, Particle swarm optimization will be used to allocate power to the subchannels depending upon the channel condition. Particle Swarm Optimization is compared with linear and root finding algorithm and it's seen that PSO provides better power allocation.

\section{Simulation and Results}

Table 1. Predefine Parameters

\begin{tabular}{|l|l|l|}
\hline Sr No. & Parameters & Values \\
\hline 1 & Number of Subcarriers & 52 \\
\hline 2 & Code rate & $1 / 2,1 / 4$ \\
\hline 3 & Modulation & $16,32,64,128,256$ \\
\hline Sr. No & Parameters & Values \\
\hline 4 & Channel & Additve White Guassian Noise \\
\hline 5 & BER & $10 \mathrm{e}-2,10 \mathrm{e}-3,10 \mathrm{e}-4,10 \mathrm{e}-5,10 \mathrm{e}-6,10 \mathrm{e}-7$ \\
\hline
\end{tabular}

Graph of OFDM system with different code rate and modulation. Input of this graph is given to Fuzzy inference system and PSO for optimization of code rate and modulation.

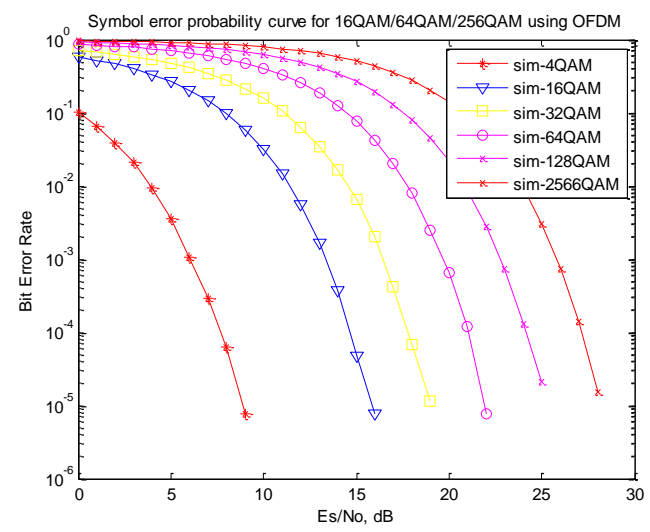

Fig.4. BER Comparison of Different QAM with Code Rate 1/2 


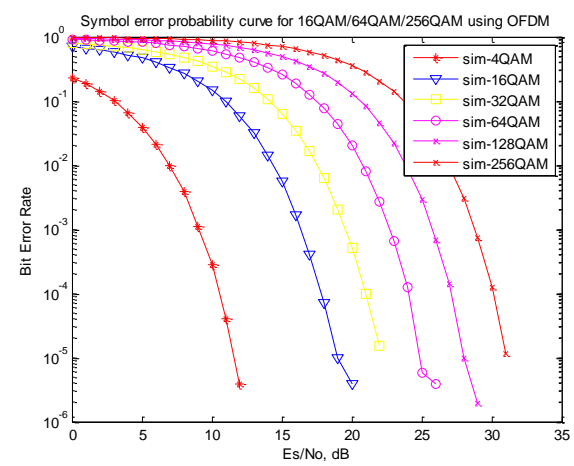

Fig.5. BER Comparison of Different QAM with Code Rate 1/4

Figure 4 and 5 shows BER performance of different QAM with respective code rate $1 / 2$ and $1 / 4$.

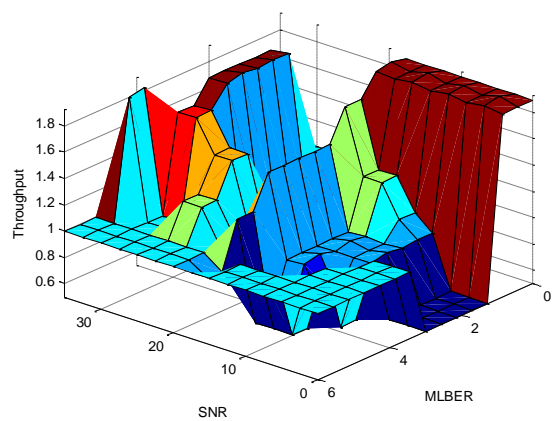

Fig.6. Fuzzy Rule Surface

Figure 6 shows the impact on throughput for different values of SNR and QoS demand after incorporating the constraint. In this diagram a higher numbered MC Pair reflects a high throughput. New MCP from FIS system is given to the transmitter in in order to improve optimization.

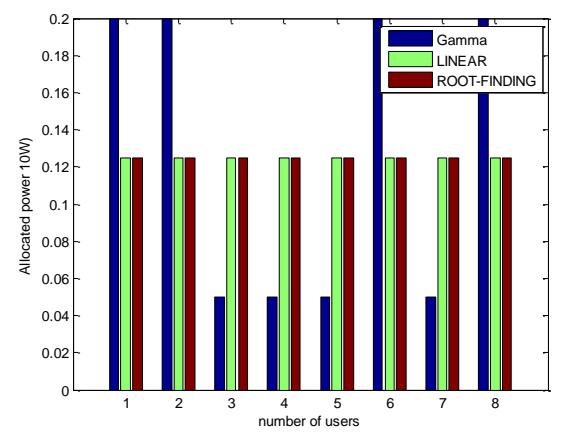

Fig.7. Power Allocation by Root Finding and Linear Method 
Figure 7 show power allocations as a function of number of users using linear and root finding. Power allocation should depend upon channel condition, but linear and root finding allocate same power to every users which leads improper communication because same power is allocated when there is less noise interference and high noise interference .

Figure 8 shows power allocation with respect to number of users using particle swarm optimization. As shown in figure 8 particle swarm optimization provide adaptive power allocation depending upon channel state condition.

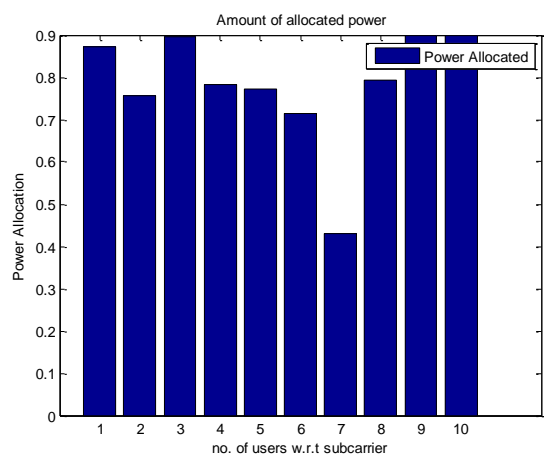

Fig.8. Power Allocation by Particle Swarm Optimization

\section{Conclusion}

FRBS and PSO are used for optimization of code rate, modulation and power respectively. PSO is used for power allocation depending upon the channel condition. After using FRBS in OFDM system it will give optimal modulation and Code rate that will have the best throughput. Thus by using FRBS and PSO optimal power allocation can be done with specified modulation techniques for specified sub channel. Comparison of power allocation for PSO is done with root finding algorithm and linear method and is seen that PSO has better power allocation.

\section{References}

[1] Y.Wu and W.Y.Zou, "Orthogonal frequency division multiplexing: A multi-carrier modulation scheme," IEEE Trans. Consumer Electronics, vol.41, no. 3, pp. 392-399, Aug. 1995.

[2] W.Y. Zou and Y.Wu, "COFDM: An overview", IEEE Trans. Broad-casting, vol.41, no. 1, pp.1-8, Mar. 1995.

[3] Derrick Wing Kwan Ng, Ernest S. Lo, and Robert Schober, "Energy-Efficient Resource Allocation in Multiuser OFDM Systems with Wireless Information and Power Transfer" University at ErlangenN"urnberg, Germany, 2010

[4] Wong, I.e.; Zukang Shen; Evans B.L., Andrews 1G., "A low complexity algorithm for proportional resource allocation in OFDMA systems”, Dept. of Electr. \& Comput. Eng., Texas Univ., Austin, TX, USA, 2004.

[5] Wong, I.e.; Zukang Shen; Evans B.L., Andrews 1G., "A low complexity algorithm for proportional resource allocation in OFDMA systems", Dept. of Electr. \& Comput. Eng., Texas Univ., Austin, TX, USA, 2004. 
Optimum Resource Allocation in Orthogonal Frequency Division Multiplexing Communication System using Fuzzy Rule Base System and Particle Swarm Optimization and Comparison with Conventional other Techniques

[6] Reddy, Y.B.; Gajendar, N.; Taylor, Portia; Madden, Damian, "Computationally Efficient Resource Allocation in OFDM Systems: Genetic Algorithm Approach", Dept of Math \& Comput. Sci., Grambling State Univ., LA, 2007, pp 36 - 41.

[7] Rajendrasingh Annauth1, Harry C.S.Rughooputh,“ OFDM Systems Resource Allocation using MultiObjective Particle Swarm Optimization”, International Journal of Computer Networks \& Communications (IJCNC) Vol.4, No.4, July 2012

[8] Atta-ur-Rahman, Qureshi I.M., Malik A.N., "A Fuzzy Rule Base Assisted Adaptive Coding and Modulation Scheme for OFDM Systems”, J. Basic Appl. Sci. Res. Vol. 2(5), pp. 4843-4853, 2012.

[9] J.Kennedy and R.C. Eberhart, "Particle Swarm Optimization", Proceedings of IEEE Conference on Neural Networks, Pages 1942 1948, 1995.

[10] S.Gheitanchi et al., "Particle Swarm Optimization for Resource Allocation in OFDMA", Proc. International Conference on digital Signal Processing, 2007.

[11] Atta-ur-Rahman, Qureshi I.M. and Muzaffar M.Z. "Adaptive Coding and Modulation for OFDM Systems using Product Codes and Fuzzy Rule Base System". International Journal of Computer Applications (IJCA), Vol. 35(4), pp. 41 -48, December 2011.

[12] Atta-ur-Rahman, Qureshi T.M., Malik A.N., "Adaptive Resource Allocation in OFDM Systems using GA and Fuzzy Rule Base System”, World Applied Sciences Journal, Vol. 1 8(6), pp. 83 6-844, 2012.

[13] Atta-ur-Rahman,“ Optimum Resource Allocation in OFDM Systems using FRBS and Particle Swarm Optimization" iBarani Institute of Information Technology, Rawalpindi, Pakistan Institute of Signals, Systems and Soft-computing (ISSS), Islamabad, Pakistan.

[14] Farhana Mustafa and Padma Lohiya. Article: Survey of Optimum Resource Allocation in OFDM Communication System. IJCA Proceedings on National Conference on Emerging Trends in Advanced Communication Technologies NCETACT 2015(4):10-13, June 2015.

\section{Author (s) Profiles}

Ms. Farhana Mustafa pursuing Master's in Communication Network from D. Y. Patil College of Engineering, Akurdi, Pune in University of Pune.

Mrs. Padma Lohiya Assistant Professor in D. Y. Patil College of Engineering, Akurdi, Pune in University of Pune.

How to cite this paper: Farhana Mustafa, Padma Lohiya,"Optimum Resource Allocation in Orthogonal Frequency Division Multiplexing Communication System using Fuzzy Rule Base System and Particle Swarm Optimization and Comparison with Conventional other Techniques", IJWMT, vol.5, no.6, pp.44-52, 2015.DOI: 10.5815/ijwmt.2015.06.06 